\title{
Effect of physical activity on body water in sedentary young underweight and obese women
}

\author{
Nasim ${ }^{4}, \mathbf{H}$.
}

\begin{abstract}
The purpose of the present study was to examine the effect of a walking exercise on body water in order to restore body water and reduction dehydration among sedentary obese and thin women. Forty young untrained girls between the age of 20-25 years (obese $\mathrm{n}=20, \mathrm{BMI}>30$ and thin $\mathrm{n}=20, \mathrm{BMI}<20$ ) volunteered to participate in this study. Participants were randomly assigned to two exercises (obese $=10$, thin $=10$ ) and two control (obese $=10$, thin=10) groups matched by BMI. DXA was used to measure each subject's body water, percentage body fat and lean mass. Serum estrogen level by radioimmunoassay (RIA) was assessed. Each walking session was $30 \mathrm{~min}$ long at an intensity corresponding to 50-75\% of maximal age adjusted heart rate, 3 days per week for 2 months. Percent body fat and lean mass were affected positively by exercise program (all $\mathrm{p}=0.000)$ and significant change was observed in Serum estrogen and body water in both exercise group $(\mathrm{p}<0.05)$. In conclusion the study demonstrated that 30 minutes walking exercise (i.e. exercise training) improved adaptation of body fluids in both obese and thin sedentary women
\end{abstract}

Keywords walking exercise, body water, obese and thin women

\section{Introduction}

During exercise, increased capillary hydrostatic pressure caused by elevation of arterial pressure produces plasma volume shifts from the vascular space to the interstitial fluids. Following a rapid efflux of vascular fluid within minutes of exercise, there is very little further reduction in plasma volume during exercise. Recall that body water content regulates sweating, and sweating provides our best method of heat loss during exercise. So, adequate body water is essential to avoid heat injury; e.g., heat exhaustion or heat stroke (Convertino, 1991). Body water retention is common in high estrogen states. There are a number of "fluid retention" hormones that exert a profound control over this fluid regulatory system. Hormones are substances in the body that act to promote certain activity by specific organs. The fluid regulatory hormones act primarily on the brain and kidney to control both intake and output of water. The most important hormone of this type is the antidiuretic hormone, which responds rapidly to changes in body water status, and is responsible for controlling the rate of water retention by the kidneys. To complicate matters, antidiuretic hormone regulation maybe modified by a variety of factors, one of which may be the female sex hormone estrogen (Akaishi \& Sakuma, 1990). Early research suggests that the greater body water content in high estrogen states

\footnotetext{
${ }^{4}$ Habibzadeh Nasim lectures at the Department of Sport Sciences, University of Guilan, Rasht, Iran.
} Email: nasim_habibzadeh@yahoo.com. 
is likely due to changes in both water intake and output in premenopausal women. In addition, fluctuations in antidiuretic hormone parallels those of estrogen throughout the menstrual cycle, and are increased following estrogen administration in postmenopausal women, suggesting that fluid retention is also likely to be increased. Thus, the combination of enhanced fluid intake and retention causes the increase in total body water content during high estrogen states. Some of this excess water is retained in the blood vessels (i.e., in the plasma), leading to plasma volume expansion, which has potent effects on physical performance during heat stress (Stachenfeld \& Taylor, 2009). Most people walk every day but it is often overlooked as an exercise activity. Walking is one of the easiest, and cheapest, ways to improve your fitness and increase specific hormones in young individual. It is a light cardiovascular exercise, which means it improves the condition of your heart and lungs (Barr, 1999). Chumlea, Schubert, Sun, Demerath, Towne, \& Siervogel, 2007) concluded that there is inadequate timely information on measured total and extra-cellular water volumes for the population. Distributional body water relationships within the body have been considered fixed, but there was evidence these relationships were affected by the level of fatness, body composition models and to our knowledge, no study has shown the effect of an exercise program on body water in obese and thin women with a mean age of 20 years simultaneously that were performing the same exercise program. The purpose of this study was to investigate whether two months program of closely supervised walking exercise would be beneficial for increasing body water among healthy sedentary obese and thin women.

\section{Material and methods}

Forty young untrained girls between the age of 20-25 years (obese $n=20$, BMI $>30$ and thin $n=20, B M I<20)$ volunteered to participate in this study. Participants were randomly assigned to two exercises (obese $=10$, thin $=10$ ) and two control (obese $=10$, thin $=10$ ) groups matched by BMI. Written informed consent for all procedures was obtained from all participants prior to entering the study and this study was approved by local Committee of Ethics. The criteria for the invitation were being willing to participate, clinically healthy (no cardiovascular, musculoskeletal, respiratory, or other chronic diseases that might limit training or testing), no menstrual irregularities, not using medication and no beta-blockers, sedentary life style (no regular sports activities for at least 2 years), non dieting, and no apparent occupational or leisure time responsibilities that could impede their participation. The following measurements were made at baseline prior to the start of the exercise program and at after completion of the 2- month training program.

\section{Anthropometric measurement}

Body weight and height were recorded and body mass index (BMI) was calculated as weight $(\mathrm{kg})$ divided by height $(\mathrm{m})$ squared. DXA (Lunar DPX-L, software version 1.31, USA) was used to measure each subject's body water, percentage body fat and lean mass. The DXA scans were performed in the Orthopaedic Diagnostic Centre at the National University Hospital, Guilan. In addition, all subjects were weighed every week.

\section{Estrogen assessment}

Blood samples were collected after an overnight fast $(>12 \mathrm{~h})$ in a sitting position and centrifuged at $1500 \mathrm{rpm}$ for 30 minutes at $4^{\circ} \mathrm{C}$ within $2 \mathrm{~h}$. Serum sample from each 
participant were stored frozen at $-20^{\circ} \mathrm{C}$ until analyzed. Serum estrogen level was assessed by radioimmunoassay (Amersham Biosciences, Piscataway, NJ, USA) in follicular stage in each subject's menstrual cycle.

\section{Exercise program}

The program included warm-up phase for 5 minutes that included stretching exercises, 30 minutes walking at 50-75\% of maximum heart rate and cooling-down phase for 5 minutes of stretching, three times a week for 2 months in indoor place. A target heart rate range between $55-70 \%$ of age adjusted maximum heart rate intensity was calculated by each walker from her age and walking supine resting heart rate (Swain, Abernathy, Smith, Lee, \& Bunn, 1994). Heart rate was measured with an electronic heart rate monitor (Sport Tester PE, Polar Electro, Oy, Finland). The exercise program was accompanied by drinking water in every 10 minutes.

\section{Data analysis}

The data were analyzed using the SPSS statistical package (SPSS 11.5 for Windows; SPSS, Chicago, IL, USA).Mean and standard deviation (SD) was used as descriptive statistic. Student's t-test was to assess the change in BMI, body weight, serum estrogen and body water before and after the exercise intervention. A significance level of set at $\mathrm{p}<0.05$ was used for all comparisons.

\section{Results}

Forty subjects $(100 \%)$ completed the training program. No major change in menstrual status was observed during the study. Table 1 shows the changes in data on both exercise and control groups. There were no significant differences in mean age, height between the groups. There is notable differences between the groups were body weight, BMI in obese and thin groups. Percent body fat and lean mass were affected positively by exercise program (all $\mathrm{p}=0.000$ ) and significant change was observed in Serum estrogen and body water in both exercise group $(\mathrm{p}<0.05)$. 
African Journal of Educational Studies in Mathematics and Sciences Vol. 8, 2010

Table1. Mean value $( \pm \mathrm{SD})$ of variables before (Pre) and (Post) training program

\begin{tabular}{|c|c|c|c|c|c|c|c|c|}
\hline \multirow{2}{*}{ Variable } & \multicolumn{2}{|c|}{ Obese(Exe) } & \multicolumn{2}{|c|}{ Obese(Con) } & \multicolumn{2}{|c|}{ Thin(Exe) } & \multicolumn{2}{|c|}{ Thin(Con) } \\
\hline & Pre & Post & Pre & Post & Pre & Post & Pre & Post \\
\hline Age (year) & $22.2 \pm 1.9$ & - & $22.6 \pm 1.5$ & - & $21.1 \pm 1.7$ & - & $21.9 \pm 1.2$ & - \\
\hline Height $(\mathrm{cm})$ & $157.7 \pm 5.1$ & - & $159.1 \pm 1.5$ & - & $159.9 \pm 7.5$ & - & $162.7 \pm 6.6$ & - \\
\hline Weight $(\mathrm{kg})$ & $74.9 \pm 8.1$ & $73.2 \pm 7.7 * * *$ & $78.1 \pm 10.8$ & $78.0 \pm 10.1$ & $45.8 \pm 5.3$ & $46.4 \pm 5.1 * * *$ & $46.4 \pm 5.7$ & $46.3 \pm 5.2$ \\
\hline BMI $\left(\mathrm{kg} / \mathrm{m}^{2}\right)$ & $30.2 \pm 1.8$ & $28.8 \pm 2.1^{* * *}$ & $30.9 \pm 3.5$ & $30.4 \pm 3.0$ & $17.7 \pm 1.0$ & $17.8 \pm 1.4$ & $17.5 \pm 1.0$ & $17.2 \pm 0.9$ \\
\hline Estrogens $(\mathrm{pg} / \mathrm{ml})$ & $30.4 \pm 15.6$ & $61.0 \pm 18.6^{* * *}$ & $23.9 \pm 13.15$ & $41.5 \pm 17.2 *$ & $25.6 \pm 8.4$ & $60.2 \pm 18.8 * * *$ & $33.0 \pm 14.3$ & $38.0 \pm 10.6$ \\
\hline Body fat content (\%) & $38.8 \pm 4$ & $36.4 \pm 6.8$ & $39.7 \pm 3.5$ & $39.0 \pm 5.2$ & $21.8 \pm 3.1$ & $20.1 \pm 3.6$ & $22.4 \pm 2.9$ & $22.4 \pm 3.2$ \\
\hline Lean body mass $(\mathrm{kg})$ & $43.3 \pm 5.3$ & $44.4 \pm 6.2$ & $43.9 \pm 6.0$ & $44.3 \pm 6.7$ & $33.5 \pm 3.7$ & $34.5 \pm 4.0 * * *$ & $33.9 \pm 4.3$ & $33.7 \pm 4.1$ \\
\hline Total Body water (L) & $30.26 \pm 78$ & $33.155 \pm 99 * * *$ & $30.11 \pm 22$ & $30.00 \pm 10$ & $19.81 \pm 9.3$ & $21.59 \pm 8.25 * * *$ & $19.18 \pm 9.3$ & $19.11 \pm 7.32$ \\
\hline
\end{tabular}

*Exe=Exercise

*Con=Control

Significantly different from the 'Pre' value:* $\mathrm{p}<0.05 ; * * *$ 


\section{Discussion}

This is the first study to assess the effect of exercise on body water in young women. This difference may be due to the younger age of subjects in our study and the nature of the mechanical load. In our patient a simple 30 minutes of walking exercise at the range of 50-75\% maximum heart rate was enough to decreasing the fat mass and increasing lean body weight. "Lean mass" means muscle."Lean mass," the researchers conclude, "is the major determinant of body size, providing further evidence that body size is adapted to the dynamic load imposed by muscle force rather than passive loading" by fat. On the other hand Muscle mass able to hold more water weight to fat mass (Thompson, Rakow $\&$ Perdue, 2004). Increase in serum estrogen and total body water in both exercise obese and thin group is good responses to walking program. These mechanisms probably include increasing plasma protein oncotic pressure, differences in peripheral vasoconstriction in active muscles and inactive tissues, and elevated lymph flow. The interaction of these factors provides optimal thermoregulatory and cardiovascular stability. The beneficial effects of hyperdydration and subsequent hypervolemia are manifest in the adaptation of body fluids and electrolytes to exercise training. Thus, with regard to fluid receiving shifts during exercise, training is an effective way to become hyper hydrated and to reduce the dehydration (Convertino, 1987). Stachenfeld \& Taylor (2009) similarly indicate water retention was the primary contributor to increasing estrogens during endurance exercise. Weinheimer, Martin, Weaver, Welch \& Campbell (2008) supported that young, physically active women can completely compensate for exercise-induced sweat losses by increasing adequate water intake. Bossingham, Carnell \& Campbell (2005) showed that healthy older adults maintain water input and balance comparable to those of younger adults and have no apparent changes in hydration status. To prevent dehydration, consuming plenty of fluids is helpful.

\section{Conclusion}

In conclusion this study this study demonstrated that 30 minutes walking exercise (i.e. exercise training) improved adaptation of body fluids in both obese and thin sedentary women.

\section{Acknowledgment}

The work was supported by grants from the University of Guilan. The authors also gratefully acknowledge the all subjects whom cooperated in this investigation.

\section{References}

Akaishi T, and Sakuma Y.(1990). Estrogen-induced modulation of hypothalamic osmoregulation in female rats. Am J Physiol. 258 (27): 924-9.

Barr SI. (1999). Effects of dehydration on exercise performance. Can J Appl Physiol. 24(2):164-72.

Bossingham MJ, Carnell NS, Campbell WW.(2005). Water balance, hydration status, and fat-free mass hydration in younger and older adults. Am J Clin Nutr. 81(6):1342-50. 
Chumlea WC, Schubert CM, Sun SS, Demerath E, Towne B, Siervogel RM.(2007). A review of body water status and the effects of age and body fatness in children and adults. J Nutr Health Aging. 2007;11(2):111-8.

Convertino VA . (1991).Blood volume: its adaptation to endurance training. Med Sci Sports Exerc. 23(12): 1338-48.

Convertino VA. (1987).Fluid shifts and hydration state: effects of long-term exercise. Can J Sport Sci. 12(l 1):136-139.

Stachenfeld N S and Taylor H S. (2009). Sex hormone effects on body fluid and sodium regulation in women with and without exercise-associated hyponatremia. $J$ Appl Physiol, 107(3): 864 - 872.

Swain DP, Abernathy KS, Smith CS, Lee SJ, Bunn SA.(1994).Target heart rates for the development of cardio respiratory fitness. Medicine and Science in sport and exercise.26 (1):112-116.

Thompson LD, Rakow J, Perdue MS. (2004) .Relationship between accumulated walking and body composition in middle-aged women. Med Sci Sports Exerc 36: 911914.

Weinheimer EM, Martin BR, Weaver CM, Welch JM, Campbell WW. (2008). The effect of exercise on water balance in premenopausal physically active women. $J$ Am Diet Assoc. 108(10):1662-7 